\title{
Gradhiva
}

GRADHIV

Revue d'anthropologie et d'histoire des arts

$9 \mid 2009$

Arts de l'enfance, enfances de l'art

\section{Emmanuel Pernoud, L'Enfant obscur. Peinture, éducation, naturalisme}

Paris, Hazan, 2007, 287 p.

\section{Claire Le Thomas}

\section{OpenEdition}

\section{Journals}

Édition électronique

URL : http://journals.openedition.org/gradhiva/1461

DOI : $10.4000 /$ gradhiva. 1461

ISSN : 1760-849X

\section{Éditeur}

Musée du quai Branly Jacques Chirac

Édition imprimée

Date de publication : 2 septembre 2009

Pagination : 204-205

ISBN : 978-2-35744-009-8

ISSN : 0764-8928

\section{Référence électronique}

Claire Le Thomas, «Emmanuel Pernoud, L'Enfant obscur. Peinture, éducation, naturalisme », Gradhiva [En ligne], 9 | 2009, mis en ligne le 02 septembre 2009, consulté le 21 septembre 2020. URL : http:// journals.openedition.org/gradhiva/1461 ; DOI : https://doi.org/10.4000/gradhiva.1461

Ce document a été généré automatiquement le 21 septembre 2020.

(c) musée du quai Branly 


\section{Emmanuel Pernoud, L'Enfant obscur. Peinture, éducation, naturalisme}

Paris, Hazan, 2007, 287 p.

Claire Le Thomas

\section{RÉFÉRENCE}

Emmanuel Pernoud, L'Enfant obscur. Peinture, éducation, naturalisme. Paris, Hazan, 2007, $287 \mathrm{p}$.

1 Après L'Invention du dessin d'enfant au début du $\mathrm{xx}^{\mathrm{e}}$ siècle $^{1}$, Emmanuel Pernoud s'attache aux représentations de l'enfant dans la peinture et la littérature du xix siècle. Richement illustré, d'une très belle mise en pages, ce livre, publié par le même éditeur, poursuit vers l'amont la réflexion menée dans le premier ouvrage. L'Enfant obscur, en décrivant la naissance d'une autre vision de l'enfant chez les artistes, explore un autre art de l'enfance dont l'apparition et le développement précèdent et accompagnent tout à la fois l'intérêt pour les productions plastiques enfantines.

2 Pour rendre compte de l'originalité des œuvres dépeignant une enfance à rebours des représentations éducatives dominantes de l'époque, l'auteur examine d'abord toute la gamme des images traditionnelles de l'enfant au XIX ${ }^{e}$ siècle. Depuis le portrait de famille jusqu'à l'imagerie laïque de l'État en passant par la peinture religieuse ou le portrait d'enfant seul, il analyse chacune de ces iconographies afin d'en expliciter le discours et l'idéologie. L'enfant y apparaît toujours encadré, sous la protection de la famille, de la religion ou de l'État, dans un milieu qui l'enserre, dessine son horizon social et l'assujettit aux valeurs spirituelles ou morales présidant à son éducation. Il est présenté comme un être apprivoisé, dégagé de la nature d'où il provient; image rassurante destinée à tempérer la part d'inconnu en lui, à faire « oublier les limbes pour insérer le nouveau venu dans un espace commun » (p. 33). C'est avec ce projet éducatif visant à donner à l'enfant un visage aimable et familier que certains artistes vont s'efforcer de rompre pour faire venir au jour une autre réalité. 
3 La première étape de ce processus de défamiliarisation, qui vise à redonner à l'enfance son opacité, consiste à mettre en scène un inconnu. La figure de l'orphelin, chez des écrivains comme Dickens, Hugo ou Balzac, coupe l'enfant des liens familiaux, l'extrait d'une ascendance quelconque et le dégage de sa position de protégé ; les portraits de Géricault montrent des enfants au regard indéchiffrable, au physique disgracieux cette macrocéphalie qui rappelle la morphologie du fœetus -, dans des postures maladroites, sur un paysage désolé. La néantisation du fond installe l'enfant dans un ailleurs, le rend étranger, tandis qu'apparaît un nouveau bestiaire qui n'évoque plus l'apprivoisement mais au contraire « rétablit l'animal dans ses fonctions de partenaire de jeu » (p.95) afin de réduire la distinction entre humanité et animalité. Dans cette entreprise de mise à distance, la dissolution du lieu est primordiale: transporter l'enfant dans un espace sans repères, non socialisé (terrain vague, route sans fin, mur aveugle, nuit...), comme le font des peintres réalistes, tels Courbet, Daumier ou Millet, revient à l'installer dans une vacance qui dément son appartenance au monde adulte. Cette enfance non située se conjugue alors avec la représentation de groupes marginalisés, exclus, errants.

4 Avec la mouvance naturaliste, la rupture se fait sentir davantage encore. La "vérité " que les naturalistes souhaitent faire apparaître, avec l'aide de la science, ne s'accorde pas avec la vision idéalisée de l'enfance mise en avant par la pensée pédagogique et son imagerie. L'objectivité du regard et du discours médical va fournir aux artistes de nouveaux moyens pour établir une distance avec leur sujet et désublimer la condition enfantine. Les nombreuses scènes d'accouchement qui empruntent leur vocabulaire au langage médical chez Zola, Maupassant, Balzac ou les Goncourt proposent une vision crue et tragique de la naissance : le nouveau-né apparait dans la douleur et le sang, il échoue dans un monde étranger, et ce drame primitif, placé sous le signe de la déchirure et de la perte, est annonciateur des naufrages à venir. Dans la littérature, le personnage de l'enfant bâtard symbolise particulièrement bien cette déchéance première ainsi que l'irréductible extériorité de l'enfant par rapport à la société qui l'accueille : sans lignée et sans liens, il apparaît comme le produit brut de la nature. La visibilité du nouveau-né et les significations qu'elle revêt - rappel de la vie prénatale, de l'inconnu d'où il provient et du tragique de la naissance, physionomie foetale loin de la ressemblance adulte - affirment que l'enfant demeure un intrus et contribuent à libérer la représentation de l'enfance des connotations de joie, de beauté, de santé ou d'amabilité qui prévalaient à l'époque. Les écrivains décrivent des enfants sales, maladifs, cruels; les peintres «n'hésitent pas à montrer ce qui peut faire peur dans le corps d'un enfant ou d'un bébé - la difformité, l'ambiguïté des sexes» (p. 123). Cette métamorphose du regard sur le corps enfantin est sensible notamment dans l'évocation de l'usure précoce provoquée par le travail, chez des peintres comme Bastien-Lepage ou des romanciers comme Zola chez qui «l'enfant devient un cas symptomatique du vieillissement accéléré des classes laborieuses » (p. 161-162). Le portrait d'enfant mort, genre qui renaît dans la seconde moitié du xix ${ }^{e}$ siècle avec la photographie mortuaire, ainsi que les récits de la mort d'un enfant chez Maupassant, Zola ou Flaubert neutralisent l'esthétisation et tuent littéralement les images traditionnelles de l'enfance. Les portraits de Manet et de Degas proposent enfin une autre démystification: l'un comme l'autre s'attachent à montrer l'artificialité de la pose et tout ce qu'elle contient de contraintes et de dressages éducatifs et esthétiques. Ils dépeignent explicitement «l'enfance dirigée » (p.177), c'est-à-dire les mécanismes de 
l'éducation et les processus d'acquisition, la manière dont les individus sont modelés et corsetés par l'hérédité et les usages.

Le naturalisme de Van Gogh est d'un autre type : il s'agit pour lui de montrer la vérité du commun, de l'insignifiant, pour atteindre la vie universelle, la complète humanité qui fait ressurgir le passé dans le présent et donne une valeur sacrée aux pratiques immémoriales. Dans cette quête, l'enfance tient une place particulière. La naissance, événement qui relève de la nature, est inscrite dans cette temporalité éternelle; elle rappelle le pouvoir rédempteur de l'enfant et possède une dimension résurrectionnelle. Elle est renaissance après une première mort : pour l'enfant qui laisse un monde pour un autre, et pour ceux qui enfantent puisqu'une part d'eux-mêmes passe dans leur progéniture. C'est donc « la vie se dépassant dans l'Éternité » (p. 241) que dépeint Van Gogh dans ses portraits d'enfants, dans ses représentations de berceaux ou de familles. Il épure ses sujets pour leur donner une apparence intemporelle; il cherche dans les visages « les secrètes déterminations qui tissent les générations entre elles, les lois de perpétuation et de transformation » (p. 234); il attribue à l'enfance une gravité et une importance bien éloignées de la puérilité et de l'insouciance qui la caractérisent généralement.

Emmanuel Pernoud revisite ainsi, à travers le prisme de l'enfance, l'œuvre de grands artistes $\mathrm{du} \mathrm{XIX}^{\mathrm{e}}$ siècle. Conjuguant peinture, littérature, traités de pédagogie et d'obstétrique et histoire sociale, dans un discours presque trop foisonnant, il propose une analyse dense d'un sujet encore peu étudié et dévoile l'importance qu'il revêt pour la compréhension des œuvres de ces écrivains et de ces peintres. Il éclaire d'un jour nouveau un corpus abondant, offrant des interprétations fines et convaincantes de nombreuses créations emblématiques de l'époque. Il livre au passage des réflexions passionnantes sur les contradictions inhérentes à l'art du portrait et fait entrevoir la portée culturelle du regard porté par ces artistes sur l'enfance. L'« état de désarmement face au motif» (p.16) dont doivent faire preuve les peintres et les écrivains pour révéler une autre vision de l'enfant évoque celui de l'ethnologue qui cherche également à se défaire des conceptions et préjugés communs pour ouvrir les yeux sur une réalité autre. La pensée développée par ces artistes entre ainsi parfois en résonance avec les études qui seront plus tard menées en anthropologie de l'enfance.

\section{NOTES}

1. Pernoud Emmanuel, L'Invention du dessin d'enfant en France, à l'aube des avant-gardes, Paris, Hazan, 2003. 